\title{
Comparison of non-coding RNAs in human and canine cancer
}

\section{Siegfried Wagner, Saskia Willenbrock, Ingo Nolte and Hugo Murua Escobar*}

Small Animal Clinic, University of Veterinary Medicine Hannover, Hannover, Germany

\section{Edited by:}

Peng Jin, Emory University School of Medicine, USA

\section{Reviewed by:}

Chris Sullivan, University of Texas at Austin, USA

Alan G. Ramsay, Queen Mary

University of London, UK

Yujing Li, Emory University, USA

*Correspondence:

Hugo Murua Escobar, Small Animal Clinic, University of Veterinary

Medicine Hannover, Buenteweg 9, 30599 Hannover, Germany.

e-mail: hescobar@tiho-hannover.de
The discovery of the post-transcriptional gene silencing (PTGS) by small non-protein-coding RNAs is considered as a major breakthrough in biology. In the last decade we just started to realize the biologic function and complexity of gene regulation by small non-coding RNAs. PTGS is a conserved phenomenon which was observed in various species such as fungi, worms, plants, and mammals. Micro RNAs (miRNA) and small interfering RNAs (siRNAs) are two gene silencing mediators constituting an evolutionary conserved class of non-coding RNAs regulating many biological processes in eukaryotes. As this small RNAs appear to regulate gene expression at translational and transcriptional level it is not surprising that during the last decade many human diseases among them Alzheimer's disease, cardiovascular diseases, and various cancer types were associated with deregulated miRNA expression. Consequently small RNAs are considered to hold big promises as therapeutic agents. However, despite of the enormous therapeutic potential many questions remain unanswered. A major critical point, when evaluating novel therapeutic approaches, is the transfer of in vitro settings to an in vivo model. Classical animal models rely on the laboratory kept animals under artificial conditions and often missing an intact immune system. Model organisms with spontaneously occurring tumors as e.g., dogs provide the possibility to evaluate therapeutic agents under the surveillance of an in intact immune system and thereby providing an authentic tumor reacting scenario. Considering the genomic similarity between canines and humans and the advantages of the dog as cancer model system for human neoplasias the analyses of the complex role of small RNAs in canine tumor development could be of major value for both species. Herein we discuss comparatively the role of miRNAs in human and canine cancer development and highlight the potential and advantages of the model organism dog for tumor research.

\section{BIOGENESIS AND FUNCTION OF SMALL RNAs IN MAMMALS}

In 2006, Andrew Fire and Craig C. Mello were awarded with the Nobel Prize in medicine for their work on RNA interference (RNAi). Since the discovery of post-transcriptional gene silencing (PTGS) mechanism in various species, the interest in using small RNA molecules and its endogenous mechanisms as a new pharmacological approach to human diseases is constantly rising (Fire et al., 1998; Elbashir et al., 2001). Micro RNAs (miRNA) and small interfering RNAs ( siRNA) are two small non-protein-coding RNA molecule types which play a leading role in PTGS. Thereby, contrary to siRNAs, miRNAs appear to act as "fine tuner" of gene regulation (Sevignani et al., 2006).

miRNAs are endogenously expressed as primary miRNA (pri-miRNA) transcripts composed of up to several thousand nucleotides (Mondol and Pasquinelli, 2012) which are processed by the nuclear enzyme Drosha to precursor miRNAs (premiRNA) (Zeng and Cullen, 2006). Following the enzymatic processing, the cytoplasmic enzyme Dicer cleaves the pre-miRNAs generating the mature double-stranded miRNAs (Bohnsack et al.,
2004; Lund et al., 2004). Dicer not only processes pre-miRNAs, it also cleaves long double stranded RNA molecules and generates the second class of small RNAs, named siRNAs, which show a miRNA-similar size of $\sim 20$ base pairs (Tomari and Zamore, 2005).

Mature miRNAs and siRNAs are chemically and physiologically indistinguishable, apparently only differing in their respective origins (Ambros et al., 2003). Further comparison of these molecules shows that the "guide strand" of miRNAs seen in mammals, is in most cases significantly but not obligatory completely complementary to the $3^{\prime}$-untranslated region of the respective target mRNA. In the case of siRNAs the "guide strand" shares absolute complementarity to a small region in the target structure. After "guide strand" incorporation into the RNAinduced silencing complex (RISC), the respective target mRNA stability and/or translation are modulated (Tomari and Zamore, 2005). Interestingly many miRNAs, their biogenesis and functions are conserved among several organisms of higher and lower complexity as fungi, worms, Drosophila, and mammals confirming the general importance of the PTGS mechanism 
(Filippov et al., 2000; Pasquinelli et al., 2000; Wu et al., 2000; Fortin et al., 2002; Yan et al., 2003; Han et al., 2004; IbanezVentoso et al., 2008).

miRNA genes itself show to be very versatile, they were described to be polycistronic or monocistronic and occasionally located in intron- as well as exon-regions of protein-coding genes. Some miRNAs are co-expressed with their target-mRNAs as one transcript (Bartel, 2004; Kim et al., 2009). Different miRNAs target a single mRNA, and a single miRNA can also be regulating the expression of many different targets. Additionally, miRNAs were also reported to be able to regulate other miRNAs by direct interactions (Winter et al., 2009; Chen et al., 2011) and in some cases miRNAs were described to be regulated by proteins translated from their respective target $\mathrm{mRNA}$, constituting a regulatory negative feedback loop (Bracken et al., 2008; Rybak et al., 2008).

In general, long double stranded RNA molecules are not common in mammals, suggesting that the RNAi mechanism mediated by siRNAs evolved for defense of viral infections and transposable elements (Obbard et al., 2009).

Despite their different origin these small non-coding RNA molecules have many things in common including the small size, specificity of inhibition, and potency and considering therapeutic applications a diminished risk to induce unspecific effects as immune responses. Due to these properties, these molecule types are considered to be potential key players in the development of next generation therapeutics for treatment of a variety of major diseases including cancer (Barh et al., 2010).

miRNA transcription and maturation is not the only process regulating functional miRNA levels. Stability of functional miRNAs is a further key factor in miRNA regulation. Molecule stability was reported to be dependent on several cis- and transacting factors varying considerably between miRNAs and their spatiotemporal expression (Kai and Pasquinelli, 2010). Exosomal release of miRNAs into the extracellular environment (Ohshima et al., 2010) and long non-coding RNAs (lncRNA) mimicking target mRNA sites and thereby acting as decoys, were also shown to decrease functional miRNA levels in cells (Cesana et al., 2011). Interestingly, numerous lncRNAs were reported to be deregulated in human cancer (Shore et al., 2012).

Typically one strand of the mature miRNA hybrid, the "guide strand" is maintained during interaction with RISC proteins while the "passenger strand" is degraded. This dichotomy is generally known to be caused by the stabilization of the "guide strand" by RISC loading, while the "passenger strand" stays unprotected (Kai and Pasquinelli, 2010). miRNA methylation, uridylation, and adenylation are some of the modifications having an influence on small RNAs half-life as well (Burroughs et al., 2010; Kai and Pasquinelli, 2010). However, miRNA stabilityenhancing proteins were also described to be actively involved in miRNA half-life, suggesting that the miRNA-mediated gene regulation processes are more complex and as variable as these genes are itself (Kai and Pasquinelli, 2010).

\section{THE DOG AS MODEL ORGANISM}

In recent years, the role of the domestic dog as model organism for various human diseases constantly gained increasing importance. Many canine inherited diseases were described, including
Alzheimer's disease (Rofina et al., 2003), narcolepsy (Lin et al., 1999), diabetes (Ionut et al., 2008), epilepsy (Lohi et al., 2005), atrial fibrillation (Shan et al., 2009), Duchenne muscular dystrophy (Mizuno et al., 2011), heart diseases (Eaton et al., 1995), and cancer (Mueller et al., 2007; Boggs et al., 2008; Noguchi et al., 2011; Uhl et al., 2011). All these disorders occur in dogs, just as in humans, spontaneously during their lifetime and many of them show similar clinical manifestations (Ostrander et al., 2000; Sutter and Ostrander, 2004).

Cancer is a complex, polygenic disease spontaneously occurring in man and dog $(\sim 1$ million diagnosed pet dog cancer cases per year in the United States), whereas tumors in most laboratory animals must be artificially induced (Mueller et al., 2007; Karlsson and Lindblad-Toh, 2008; Paoloni and Khanna, 2008). Indeed, mans best friend shares many features, including tumor genetics, molecular targets, histological appearance, and response to conventional therapies (Paoloni and Khanna, 2008). Additionally dogs often cohabitate with their owners, are exposed to similar environmental stresses, which may have a big impact on cancer development, and enjoy the best medical care among pets (Sutter and Ostrander, 2004; Rowell et al., 2011). Furthermore, dogs show a higher genetic variability than inbred laboratory mice and enable an easier and faster surgical intervention and imaging due to body size (Mueller et al., 2007). The five- to eight-fold faster aging of dogs in comparison to humans facilitates longterm studies of cancer treatments (Rowell et al., 2011). In 2005, the sequenced canine genome was published (Lindblad-Toh et al., 2005). Having a less different genome from humans than rodents and sharing a similar metabolism, according to their body size, the dog classifies as a very good model organism for molecular studies on human diseases (Sutter and Ostrander, 2004; Karlsson and Lindblad-Toh, 2008; Pinho et al., 2012).

In contrast to investigations on human miRNAs in cancer, the research on canine miRNAs is often limited by the lack of specific canine assays. Up to now only a limited number of studies were done on canine non-neoplastic and tumor tissues. In 2008, Zhou et al. identified 357 canine miRNA-genes by computational analysis, 300 of these were homologs of known human miRNAs (Zhou et al., 2008). Currently 1527 human and 323 canine miRNA matches of hairpin precursors are registered in the webdatabase, miRBase (Sanger Institute, version 16.0) (Kozomara and Griffiths-Jones, 2011).

Due to high homology of mature miRNAs between human and dog (Table 1), many of the human miRNA assays can be used for analyses of canine miRNA expression. In Table 1 canine miRNAs are listed, which share absolute homology to the human counterparts. Homologous canine miRNAs with overhangs or major sequential differences are not listed.

\section{COMPARATIVE MIRNA EXPRESSION IN HUMAN AND CANINE DISEASES}

Humans share many diseases with their canine companions including atrial fibrillation, Duchenne muscular dystrophy, and cancer, but the number of comparative studies, focussing on the role of miRNAs in canine diseases, is still relatively low (Karlsson and Lindblad-Toh, 2008; Shan et al., 2009; Mizuno et al., 2011). However, the published data is constantly growing 
Table 1 | Comparison of canine and human mature miRNAs.

\begin{tabular}{|c|c|}
\hline Canine mature miRNA & Human mature miRNA \\
\hline cfa-miR-1 & hsa-miR-1 \\
\hline cfa-let-7a & hsa-let-7a \\
\hline cfa-let-7b & hsa-let-7b \\
\hline cfa-let-7c & hsa-let-7c \\
\hline cfa-let-7e & hsa-let-7e \\
\hline cfa-let-7f & hsa-let-7f-5p \\
\hline cfa-let-7g & hsa-let-7g-5p \\
\hline cfa-miR-7 & hsa-miR-7-5p \\
\hline cfa-miR-9 & hsa-miR-9-5p \\
\hline cfa-miR-10 & hsa-miR-10a-5p \\
\hline cfa-miR-15a & hsa-miR-15a-5p \\
\hline cfa-miR-15b & hsa-miR-15b-5p \\
\hline cfa-miR-16 & hsa-miR-16-5p \\
\hline cfa-miR-17 & hsa-miR-17-3p \\
\hline cfa-miR-18a & hsa-miR-18a-5p \\
\hline cfa-miR-18b & hsa-miR-18b-5p \\
\hline cfa-miR-19a & hsa-miR-19a-3p \\
\hline cfa-miR-19b & hsa-miR-19b-3p \\
\hline cfa-miR-20a & hsa-miR-20a-5p \\
\hline cfa-miR-20b & hsa-miR-17-5p \\
\hline cfa-miR-21 & hsa-miR-21-5p \\
\hline cfa-miR-22 & hsa-miR-22-3p \\
\hline cfa-miR-23a & hsa-miR-23a-3p \\
\hline cfa-miR-23b & hsa-miR-23b-3p \\
\hline cfa-miR-25 & hsa-miR-25-3p \\
\hline cfa-miR-26a & hsa-miR-26a-5p \\
\hline cfa-miR-27a & hsa-miR-27a-3p \\
\hline cfa-miR-27b & hsa-miR-27b-3p \\
\hline cfa-miR-28 & hsa-miR-28-3p \\
\hline cfa-miR-29a & hsa-miR-29a-3p \\
\hline cfa-miR-29b & hsa-miR-29b-3p \\
\hline cfa-miR-29c & hsa-miR-29c-3p \\
\hline cfa-miR-30b & hsa-miR-30b-5p \\
\hline cfa-miR-30e & hsa-miR-30e-3p \\
\hline cfa-miR-33 & hsa-miR-33a-5p \\
\hline cfa-miR-34a & hsa-miR-34a-5p \\
\hline cfa-miR-34c & hsa-miR-34c-5p \\
\hline cfa-miR-92a & hsa-miR-92a-3p \\
\hline cfa-miR-92b & hsa-miR-92b-3p \\
\hline cfa-miR-93 & hsa-miR-93-5p \\
\hline cfa-miR-95 & hsa-miR-95 \\
\hline cfa-miR-96 & hsa-miR-96-5p \\
\hline cfa-miR-98 & hsa-miR-98 \\
\hline cfa-miR-99a & hsa-miR-99a-5p \\
\hline cfa-miR-99b & hsa-miR-99b-5p \\
\hline cfa-miR-101 & hsa-miR-101-3p \\
\hline cfa-miR-103 & hsa-miR-103a-3p \\
\hline cfa-miR-105a & hsa-miR-105-5p \\
\hline cfa-miR-106a & hsa-miR-17-5p \\
\hline cfa-miR-106a & hsa-miR-106a-5p \\
\hline cfa-miR-106b & hsa-miR-106b-5p \\
\hline
\end{tabular}

Table 1 | Continued

Canine mature miRNA

Human mature miRNA

cfa-miR-107

cfa-miR-122

cfa-miR-125a

cfa-miR-125b

cfa-miR-126

cfa-miR-127

cfa-miR-128

cfa-miR-129

cfa-miR-130a

cfa-miR-130b

cfa-miR-133b

cfa-miR-133c

cfa-miR-134

cfa-miR-135a-5p

cfa-miR-135b

cfa-miR-136

cfa-miR-137

cfa-miR-138a

cfa-miR-143

cfa-miR-145

cfa-miR-146a

cfa-miR-146b

cfa-miR-147

cfa-miR-148a

cfa-miR-148b

cfa-miR-149

cfa-miR-150

cfa-miR-151

cfa-miR-152

cfa-miR-153

cfa-miR-155

cfa-miR-181a

cfa-miR-181b

cfa-miR-181d

cfa-miR-182

cfa-miR-183

cfa-miR-184

cfa-miR-185

cfa-miR-186

cfa-miR-187

cfa-miR-190a

cfa-miR-190b

cfa-miR-191

cfa-miR-192

cfa-miR-193a

cfa-miR-193b

cfa-miR-194

cfa-miR-196a

cfa-miR-197

cfa-miR-199

cfa-miR-200a
hsa-miR-107

hsa-miR-122-5p

hsa-miR-125a-5p

hsa-miR-125b-5p

hsa-miR-126-5p

hsa-miR-127-3p

hsa-miR-128

hsa-miR-129-5p

hsa-miR-130a-3p

hsa-miR-130b-3p

hsa-miR-133b

hsa-miR-133a

hsa-miR-134

hsa-miR-135a-5p

hsa-miR-135b-5p

hsa-miR-136-5p

hsa-miR-138-5p

hsa-miR-143-3p

hsa-miR-145-5p

hsa-miR-146a-5p

hsa-miR-146b-5p

hsa-miR-147b

hsa-miR-148a-3p

hsa-miR-148b-3p

hsa-miR-149-5p

hsa-miR-150-5p

hsa-miR-151a-5p

hsa-miR-152

hsa-miR-153

hsa-miR-155-5p

hsa-miR-181a-5p

hsa-miR-181b-5p

hsa-miR-181d

hsa-miR-182-5p

hsa-miR-183-5p

hsa-miR-184

hsa-miR-185-5p

hsa-miR-186-5p

hsa-miR-187-3p

hsa-miR-190a

hsa-miR-190b

hsa-miR-191-5p

hsa-miR-192-5p

hsa-miR-193a-5p

hsa-miR-193b-5p

hsa-miR-194-5p

hsa-miR-196a-5p

hsa-miR-197-3p

hsa-miR-199a-3p

hsa-miR-200a-5p
hsa-miR-137 
Table 1 | Continued

\section{Canine mature miRNA}

cfa-miR-200b

cfa-miR-200c

cfa-miR-202

cfa-miR-203

cfa-miR-204

cfa-miR-205

cfa-miR-206

cfa-miR-208a

cfa-miR-208b

cfa-miR-212

cfa-miR-214

cfa-miR-216a

cfa-miR-216b

cfa-miR-218

cfa-miR-219

cfa-miR-219*

cfa-miR-221

cfa-miR-222

cfa-miR-223

cfa-miR-299

cfa-miR-301a

cfa-miR-301b

cfa-miR-302a

cfa-miR-302c

cfa-miR-302d

cfa-miR-320

cfa-miR-323

cfa-miR-324

cfa-miR-326

cfa-miR-328

cfa-miR-329b

cfa-miR-330

cfa-miR-331

cfa-miR-335

cfa-miR-338

cfa-miR-33b

cfa-miR-340

cfa-miR-342

cfa-miR-346

cfa-miR-361

cfa-miR-362

cfa-miR-365

cfa-miR-367

cfa-miR-370

cfa-miR-374a

cfa-miR-374b

cfa-miR-375

cfa-miR-376a

cfa-miR-376b

cfa-miR-377

cfa-miR-379
Human mature miRNA

hsa-miR-200b-5p

hsa-miR-200c-3p

hsa-miR-202-5p

hsa-miR-203a

hsa-miR-204-5p

hsa-miR-205-5p

hsa-miR-206

hsa-miR-208a

hsa-miR-208b

hsa-miR-212-5p

hsa-miR-214-3p

hsa-miR-216a-5p

hsa-miR-216b

hsa-miR-218-5p

hsa-miR-219-5p

hsa-miR-219-2-3p

hsa-miR-221-3p

hsa-miR-222-3p

hsa-miR-223-3p

hsa-mir-299

hsa-miR-301a-3p

hsa-miR-301b

hsa-miR-302a-5p

hsa-miR-302c-5p

hsa-miR-302d-5p

hsa-miR-320a

hsa-miR-323a-3p

hsa-miR-324-5p

hsa-miR-326

hsa-miR-328

hsa-miR-329

hsa-miR-330-5p

hsa-miR-331-3p

hsa-miR-335-5p

hsa-miR-3065-5p

hsa-miR-33b-5p

hsa-miR-340-5p

hsa-miR-342-3p

hsa-miR-346

hsa-miR-361-5p

hsa-miR-362-5p

hsa-miR-365a-3p

hsa-miR-367-5p

hsa-miR-370

hsa-miR-374a-5p

hsa-miR-374b-5p

hsa-miR-375

hsa-miR-376a-3p

hsa-miR-376b-3p

hsa-miR-377-5p

hsa-miR-379-5p

Table 1 | Continued

Canine mature miRNA

cfa-miR-381

cfa-miR-383

cfa-miR-410

cfa-miR-421

cfa-miR-423a

cfa-miR-424

cfa-miR-425

cfa-miR-432

cfa-miR-433

cfa-miR-448

cfa-miR-449

cfa-miR-450b

cfa-miR-451

cfa-miR-452

cfa-miR-454

cfa-miR-455

cfa-miR-487a

cfa-miR-487b

cfa-miR-488

cfa-miR-489

cfa-miR-490

cfa-miR-491

cfa-miR-493

cfa-miR-494

cfa-miR-495

cfa-miR-496

cfa-miR-497

cfa-miR-499

cfa-miR-500

cfa-miR-504

cfa-miR-505

cfa-miR-532

cfa-miR-539

cfa-miR-542

cfa-miR-543

cfa-miR-544

cfa-miR-551a

cfa-miR-551b

cfa-miR-568

cfa-miR-574

cfa-miR-590

cfa-miR-599

cfa-miR-628

cfa-miR-652

cfa-miR-660

cfa-miR-671

cfa-miR-708

cfa-miR-758

cfa-miR-761

cfa-miR-802

cfa-miR-874
Human mature miRNA

hsa-miR-381-3p

hsa-miR-383

hsa-miR-410

hsa-miR-421

hsa-miR-423-5p

hsa-miR-424-3p

hsa-miR-425-5p

hsa-miR-432-5p

hsa-miR-433

hsa-miR-448

hsa-miR-449a

hsa-miR-450b-5p

hsa-miR-451a

hsa-miR-452-5p

hsa-miR-454-3p

hsa-miR-455-5p

hsa-miR-487a

hsa-miR-487b

hsa-miR-488-5p

hsa-miR-489

hsa-miR-490-3p

hsa-miR-491-3p

hsa-miR-493-3p

hsa-miR-494

hsa-miR-495-3p

hsa-miR-496

hsa-miR-497-5p

hsa-miR-499a-5p

hsa-miR-500a-3p

hsa-miR-504

hsa-miR-505-5p

hsa-miR-532-5p

hsa-miR-539-5p

hsa-miR-542-3p

hsa-miR-543

hsa-miR-544a

hsa-miR-551a

hsa-miR-551b-3p

hsa-miR-568

hsa-miR-574-3p

hsa-miR-590-3p

hsa-miR-599

hsa-miR-628-5p

hsa-miR-652-3p

hsa-miR-660-5p

hsa-miR-671-3p

hsa-miR-708-5p

hsa-miR-758-3p

hsa-miR-761

hsa-miR-802

hsa-miR-874 
Table 1 | Continued

\begin{tabular}{ll}
\hline Canine mature miRNA & Human mature miRNA \\
\hline cfa-miR-875 & hsa-miR-875-5p \\
cfa-miR-876 & hsa-miR-876-5p \\
cfa-miR-885 & hsa-miR-885-5p \\
cfa-miR-1306 & hsa-miR-1306-5p \\
cfa-miR-1307 & hsa-miR-1307-3p \\
\hline
\end{tabular}

207 of the canine mature miRNAs listed in miRBase (Sanger Institute, version 16.0) show absolute sequence complementarity to the human counterparts. The sequence identity of the canine mature miRNA sequences with the corresponding human homologs was confirmed by miRBase, single sequence search (http://www.mirbase.org/search.shtml).

and thus it is likely that in future miRNA studies on the canine model, like in the following examples, will gain more importance.

\section{miRNAs IN NON-NEOPLASTIC DISEASES}

Recent studies showed that nicotine can induce atrial structural remodeling and increase atrial fibrosis vulnerability in dogs. Shan et al. reported a decreased miR-133 and miR-590 expression in smoking individuals with atrial fibrosis and showed that an ectopic over-expression of $m i R-133$ and $m i R-590$ resulted in a post-transcriptional suppression of TGF- $\beta 1$ and TGF- $\beta$ RII in cultured canine atrial fibroblasts (Shan et al., 2009).

Another disease affecting man as well as dogs is Duchenne muscular dystrophy. It is a lethal X-chromosome linked disorder caused by mutations in the dystrophin gene, which encodes a cytoskeletal protein. Mizuno et al. studied serum miRNA expression in the X-linked muscular dystrophy in Japan dog model $\left(\mathrm{CXMD}_{\mathrm{J}}\right)$ and found, as in humans, increased miR-1, miR-133a, and miR-206 levels (Cacchiarelli et al., 2010, 2011; Mizuno et al., 2011). The study indicates that serum miRNAs might be a reliable biomarker for muscular dystrophy (Mizuno et al., 2011).

\section{miRNAs IN CANCER}

Focusing cancer in more detail, deregulated miRNA expression was associated with many human and canine neoplasias (Mueller et al., 2007; Barh et al., 2010; Noguchi et al., 2011; Uhl et al., 2011). As miRNAs are involved in a variety of biological processes as regulation of apoptosis, angiogenesis, cell cycle control, and cell migration it is not surprising that these molecules show an enormous influence on cancer etiology (Bueno and Malumbres, 2011; Donnem et al., 2012; Landskroner-Eiger et al., 2012). For example the human miR-17-92 cluster coded miRNAs where reported to act tumorigenic, while others such as the let-7 family members, where reported to be like a coin with two sides, acting in some cases as tumor suppressors or promoting tumor development (Blenkiron and Miska, 2007; Boyerinas et al., 2010; Olive et al., 2010; Ryland et al., 2012).

In cancer, miRNA target sites and miRNA genes itself were found to be directly mutated or their expression deregulated by other factors (Ikeda et al., 2011; Ryland et al., 2012). Due to the complex acting and regulation mechanisms it is very likely that many miRNA deregulations associated with their respective disease are not even identified.

However, despite of the fact that the detailed mechanisms of miRNA action are still under debate, many diagnostic and therapeutic miRNA-based approaches show promising results (Li et al., 2009; Krell et al., 2012).

As in humans, in dogs, many miRNAs are conserved emphasizing the role of the domestic dog as model organism for miRNA in cancer research. It is very likely that these molecules also follow comparable expression patterns and similar function in canine neoplasias. The analysis of miRNA biogenesis and expression pattern could decipher the role of human and canine miRNAs in cancer and enable the design of new therapies based on small RNA delivery.

\section{miRNAs in mammary tumors}

Mammary tumors are among the most common neoplasias of female dogs, with an estimated lifetime risk for malignant tumors varying from 2 to more than 20\%. The risk for malignant mammary tumors in dogs spayed before and after their first estrus, is in comparison to intact dogs 0.05 and $8 \%$. In dogs spayed after their second estrus, the risk rises up to $26 \%$ (Withrow and Vail, 2007). Data from a Swedish study, based on 80,000 insured female, mostly sexually intact dogs, reported a rate of 111 mammary tumors (benign and malignant) per 10,000 dog-years risk (Egenvall et al., 2005). The age-standardized incidence rate for human breast cancer estimates 66.4 per 100,000 in more developed areas and is thus the most common cancer (Jemal et al., 2011).

According to a recent study, nine miRNAs, let-7f, miR-15a, miR-16, miR-17-5p, miR-21, miR-29b, miR-125b, miR-155, and $m i R-181 b$ involved in human mammary cancer, appear to follow the same expression pattern in the canine counterpart. In this study, only the investigated $m i R-145$ was not shown to be differently expressed comparing non-neoplastic and neoplastic canine tissues (Boggs et al., 2008).

\section{miRNAs linked to lymphomas}

Besides mammary cancer, canine lymphomas show the highest estimated annual incidence with 13 to 24 cases per 100,000 accounting for up to $24 \%$ of all canine neoplasias (Withrow and Vail, 2012). In human, chronic lymphocytic leukemia (CLL) is the most common leukemia in the Western world with an annual incidence of approximately 10,000 new cases in the United States (Calin and Croce, 2009).

In a recent study, the relative expression pattern of 12 canine miRNAs (cfa-let-7a, cfa-miR-15a, cfa-miR-16, cfa-miR-17-5p, cfamiR-21, cfa-miR-26b, cfa-miR-29b, cfa-miR-125b, cfa-miR-150, $c f a-m i R-155, c f a-m i R-181 a$, and $c f a-m i R-223)$ in CLL was analyzed. Due to stable expression between the investigated samples four of the 12 miRNAs (cfa-let-7a, cfa-miR-17-5p, cfa-miR-26b and $c f a-m i R-223$ ) were used as endogenous controls. miR-15a, $m i R-16$, and $m i R-181 a$ were reported to be downregulated in canine and human CLL (Calin et al., 2002; Gioia et al., 2011; Zhu et al., 2012). Four of the investigated miRNAs (cfa-miR21, cfa-miR-125b, cfa-miR-150, cfa-miR-155) were described to be upregulated in human and canine B-CLL as well (Pekarsky et al., 
2006; Fulci et al., 2007; Wang et al., 2008; Bousquet et al., 2010; Palamarchuk et al., 2010; Rossi et al., 2010). Only miR-29b, which was shown to be downregulated in human B-CLL, was upregulated in canine CLL (Pekarsky et al., 2006; Fulci et al., 2007; Wang et al., 2008; Bousquet et al., 2010; Palamarchuk et al., 2010; Rossi et al., 2010).

It was also observed by Gioa et al. that in comparison to canine B-CLL the miR-125 expression was significantly downregulated in canine T-CLL. On the basis of the mature miRNA expression ratio between $m i R-150 / m i R-125 b$, and $m i R-150 / m i R-155$, it was reported that it is also possible to distinguish among normal blood, B-CLL and T-CLL samples (Gioia et al., 2011). This illustrates the potential of miRNA expression analyses not solely as tumor marker but as an instrument to distinguish between different but similar cell or cancer types.

\section{miRNAs associated with melanomas}

Melanomas are very aggressive malignant skin cancers in man and dog (Noguchi et al., 2011). Accounting for 5-7\% of canine skin tumors. Tumors of the melanocytes and melanoblasts are relatively common in dogs (Withrow and Vail, 2007; Uhl et al., 2011). In human melanoma cell lines A2058, Mewo, and canine melanoma LMeC cells as well as malignant melanoma tissues the miR-145, miR-203, and miR-205 expression was reported to be downregulated. An ectopic expression of each of these miRNAs-induced in vitro growth inhibition in A2058, Mewo, and LMeC cells (Noguchi et al., 2011, 2012a,b), indicating their potential for treatment of human and canine malignant melanoma.

\section{miRNAs involved in epithelial to mesenchymal transition}

Furthermore, aberrant activation of the epithelial to mesenchymal transition (EMT) has been observed to promote invasion and metastasis in several human cancers. The EMT inducers ZEB1 and ZEB2 have been shown to be direct targets of the miR-200 family (miR-200a, miR-200b, miR-200c, miR-141, and $m i R-429)$ in the human breast cancer cell line MDA-MB-231 and in Madin Darby canine kidney epithelial cells (MDCK). Lost expression of these miRNAs was detected in human metaplastic breast cancer specimens, indicating that downregulation of miR-200 family members may be an important step in tumor progression (Bracken et al., 2008; Gregory et al., 2008; Adam et al., 2009).

\section{miRNAs with prognostic significance in osteosarcoma}

Representing 1\% of diagnosed cancer cases in the United States, osteosarcoma is one of the most common primary malignancies of human bone in children and adolescents (Mirabello et al., 2009). Estimated at $>10,000$ cases per year, canine osteosarcoma is relatively common in contrast to humans. Like in man, the canine counterpart also arises spontaneously in dogs and shows similar anatomical and functional biology (Khanna et al., 2006; Sarver et al., 2013). Recently Sarver et al. demonstrated an inverse correlation between human 14q32 cluster miRNA expression and aggressive tumor behavior in human and canine osteosarcoma. The group mapped the $14 \mathrm{q} 32$ cluster to the canine genome. The miR-134 and miR-544 of the 14q32 cluster, showing 100\% homology between both species, were used to examine the expression in canine samples. They showed a lower $m i R-134$ and $m i R-544$ expression in canine and human bone tumors in comparison to healthy tissues (Sarver et al., 2013). The expression levels of these two miRNAs could provide prognostic utility in osteosarcoma, a disease that shows conserved features between human and dog (Sarver et al., 2013).

For a better overview the previously described miRNA expression patterns in the different canine and human neoplasias were summarized in the Table 2. The described results should be considered with care as major differences could be present in the comparison depending on species, organism the system of tumor

Table 2 | Overview of the above described miRNAs involved in canine and human cancer.

\begin{tabular}{|c|c|c|c|}
\hline \multicolumn{4}{|c|}{ MAMMARY TUMORS } \\
\hline cfa-let-7f & $\uparrow$ & hsa-let-7f-5p & $\uparrow$ \\
\hline cfa-miR-15a & $\downarrow$ & hsa-miR-15a-5p & $\downarrow$ \\
\hline cfa-miR-16 & $\downarrow$ & hsa-miR-16-5p & $\downarrow$ \\
\hline cfa-miR-17-5p & $\downarrow$ & hsa-miR-17-5p & $\downarrow$ \\
\hline cfa-miR-21 & $\uparrow$ & hsa-miR-21-5p & $\uparrow$ \\
\hline cfa-miR-29b & $\uparrow$ & hsa-miR-29b-3p & $\uparrow$ \\
\hline cfa-miR-125b & $\downarrow$ & hsa-miR-125b-5p & $\downarrow$ \\
\hline cfa-miR-145 & $=$ & hsa-miR-145-5p & $\downarrow$ \\
\hline cfa-miR-155 & $\downarrow$ & hsa-miR-155-5p & $\downarrow$ \\
\hline cfa-miR-181b & $\uparrow$ & hsa-miR-181b-5p & $\uparrow$ \\
\hline \multicolumn{4}{|l|}{ EMT } \\
\hline cfa-miR-141 & $\downarrow$ & hsa-miR-141-3p & $\downarrow$ \\
\hline cfa-miR-200a & $\downarrow$ & hsa-miR-200a-5p & $\downarrow$ \\
\hline cfa-miR-200b & $\downarrow$ & hsa-miR-200b-5p & $\downarrow$ \\
\hline cfa-miR-200c & $\downarrow$ & hsa-miR-200c-5p & $\downarrow$ \\
\hline cfa-miR-429 & $\downarrow$ & hsa-miR-429 & $\downarrow$ \\
\hline \multicolumn{4}{|l|}{ B-CLL/T-CLL } \\
\hline cfa-miR-15a & $\downarrow / \downarrow$ & hsa-miR-15a-5p & $\downarrow /-$ \\
\hline cfa-miR-16 & $\downarrow / \downarrow$ & hsa-miR-16-5p & $\downarrow /-$ \\
\hline cfa-miR-21 & $\uparrow / \uparrow$ & hsa-miR-21-5p & $\uparrow /-$ \\
\hline cfa-miR-29b & $\uparrow / \uparrow$ & hsa-miR-29b & $\downarrow /-$ \\
\hline cfa-miR-125b & $\uparrow / \downarrow$ & hsa-miR-125b & $\uparrow /-$ \\
\hline cfa-miR-150 & $\uparrow / \uparrow$ & hsa-miR-150-5p & $\uparrow /-$ \\
\hline cfa-miR-155 & $\uparrow / \uparrow$ & hsa-miR-155-5p & $\uparrow /-$ \\
\hline cfa-miR-181a & $\downarrow / \downarrow$ & hsa-miR-181a-5p & $\downarrow /-$ \\
\hline \multicolumn{4}{|l|}{ MELANOMA } \\
\hline cfa-miR-145 & $\downarrow$ & hsa-miR-145-5p & $\downarrow$ \\
\hline cfa-miR-203 & $\downarrow$ & hsa-miR-203a & $\downarrow$ \\
\hline cfa-miR-205 & $\downarrow$ & hsa-miR-205-5p & $\downarrow$ \\
\hline \multicolumn{4}{|c|}{ OSTEOSARCOMA } \\
\hline cfa-miR-134 & $\downarrow$ & hsa-miR-134 & $\downarrow$ \\
\hline cfa-miR-544 & $\downarrow$ & hsa-miR-544a & $\downarrow$ \\
\hline
\end{tabular}

In the 1st column are the canine and in the 3rd the human miRNAs listed. In the 2 nd and 4th column the relative expression or tendencies in comparison to non-neoplastic samples are presented. "-" indicates that no reports were found for involvement in CLL. "=" means that no differences in expression between tumor and healthy cells were described. " $\uparrow$ " signifies upregulation or " $\downarrow$ " downregulation in comparison to non-neoplastic samples. 
classification, type of miRNA preparation and quantification and the used type of normalization.

\section{FUTURE PROSPECTS}

As the majority of miRNAs involved in human and canine diseases are evolutionary conserved, it is likely that the expression patterns are also similar. Nevertheless homologous miRNAs, showing similar pattern of expression in different species, should be considered with care as it is possible that the functions still deviate. Even individual miRNAs in the same species can show oncogene suppressive functions or act oncogenic (Boggs et al., 2008).

However, some miRNAs were shown to be a potential noninvasive biomarker for different clinically relevant subtypes of human breast cancer (Cortez et al., 2012; Shore et al., 2012). As aberrant miRNA expression is partially postulated to be an early event in human tumorigenesis (Cortez et al., 2012) it is tempting to speculate that specific miRNAs could also be used as prognostic tools (Li et al., 2009; Krell et al., 2012) for canine neoplasias and thus should be further evaluated as novel agents in the future.

Further knowledge of spatiotemporal miRNA expression and their respective targets will allow more specific modulation of target or effector molecule expression by delivery of miRNAs, siRNAs, or similar modified oligonucleotides.

A directed ectopic expression of naturally occurring miRNAs could have the capability to act therapeutically in an organism by replenishing the missing tumor suppressor miRNA and interfering with oncogenic properties of cancer cells. In perspective oncomiRs (cancer-promoting miRNAs) could be suppressed by antagomiRs (chemically engineered oligonucleotides that act as miRNA inhibitors) or functionally inhibited by titering them away with IncRNAs (Cesana et al., 2011). Due to the fact that a

\section{REFERENCES}

Adam, L., Zhong, M., Choi, W., Qi, W., Nicoloso, M., Arora, A., et al. (2009). miR-200 expression regulates epithelial-to-mesenchymal transition in bladder cancer cells and reverses resistance to epidermal growth factor receptor therapy. Clin. Cancer Res. 15, 5060-5072.

Ambros, V., Bartel, B., Bartel, D. P., Burge, C. B., Carrington, J. C., Chen, X., et al. (2003). A uniform system for microRNA annotation. RNA 9, 277-279.

Barh, D., Malhotra, R., Ravi, B., and Sindhurani, P. (2010). MicroRNA let-7: an emerging next-generation cancer therapeutic. Curr. Oncol. 17, 70-80.

Bartel, D. P. (2004). MicroRNAs: genomics, biogenesis, mechanism, and function. Cell 116, 281-297.

Blenkiron, C., and Miska, E. A. (2007). miRNAs in cancer: approaches, aetiology, diagnostics and therapy. Hum. Mol. Genet. 16, R106-R113.

Boggs, R. M., Wright, Z. M., Stickney, M. J., Porter, W. W., and
Murphy, K. E. (2008). MicroRNA expression in canine mammary cancer. Mamm. Genome 19, 561-569.

Bohnsack, M. T., Czaplinski, K., and is a RanGTP-dependent dsRNAbinding protein that mediates nuclear export of pre-miRNAs. RNA 10, 185-191. B., and Lodish, H. F. (2010). MicroRNA miR-125b causes leukemia. Proc. Natl. Acad. Sci. U.S.A. 107, 21558-21563.

Boyerinas, B., Park, S. M., Hau, A., (2010). The role of let-7 in cell differentiation and cancer. Endocr. Relat. Cancer 17, F19-F36.

Bracken, C. P., Gregory, P. A., Kolesnikoff, N., Bert, A. G., Wang, J., Shannon, M. F., et al. (2008). A double-negative feedback loop between ZEB1-SIP1 and the microRNA-200 family regulates epithelial-mesenchymal transition. Cancer Res. 68, 7846-7854. Gorlich, D. (2004). Exportin 5

Bousquet, M., Harris, M. H., Zhou, Murmann, A. E., and Peter, M. E.

single miRNA can act on several targets, a miRNA-based therapy could have significant advantages but also bears the risk to induce unintended side effects. Thus, modifications of gene expression by more stringent artificial miRNAs or siRNAs sharing $100 \%$ homology to a single target of interest could lower the risk for off target effects, improve treatment, and reduce unwanted side effects.

However, two major obstacles still remain: intracellular delivery and expression level. The ectopic expressed miRNAs must show a certain expression level to reconstitute the "normal" state of genes and the applied small RNAs must be taken up by cancer cells and be further correctly incorporated into RISC. Until now, multiple delivery strategies such as nanoparticles, liposomes, peptide nucleic acids, and viral vectors have been described to achieve this goal but none of these can be used ubiquitously for different types of neoplasias in different locations (Petrocca and Lieberman, 2010; Pan et al., 2011).

Showing many advantages concerning specificity, potency, number of accessible targets, species cross-reactivity, fast development and the scalability, small RNAs may have an enormous diagnostic and therapeutic potential in cancer treatment (Li et al., 2009; Krell et al., 2012) as single agents or e.g., substituting antibody-based cancer therapies.

Homology between human and canine miRNAs could not only enable to use the dog as model organism, but also the transfer of therapeutic and diagnostic approaches established for humans to canines and vice versa. Further elucidation of miRNA functions and biogenesis will facilitate and improve the design and entry of small RNA therapeutic programs into clinical practice. Until now only a few studies describe miRNA expression in canines. Thus, a systematic profiling of miRNA expression would be of great value.

Bueno, M. J., and Malumbres, M. (2011). MicroRNAs and the cell cycle. Biochim. Biophys. Acta 1812, 592-601.

Burroughs, A. M., Ando, Y., De Hoon, M. J., Tomaru, Y., Nishibu, T., Ukekawa, R., et al. (2010). A comprehensive survey of 3' animal miRNA modification events and a possible role for 3' adenylation in modulating miRNA targeting effectiveness. Genome Res. 20, 1398-1410.

Cacchiarelli, D., Legnini, I., Martone, J., Cazzella, V., D’Amico, A., Bertini, E., et al. (2011). miRNAs as serum biomarkers for Duchenne muscular dystrophy. EMBO Mol. Med. 3, 258-265.

Cacchiarelli, D., Martone, J., Girardi, E., Cesana, M., Incitti, T., Morlando, M., et al. (2010). MicroRNAs involved in molecular circuitries relevant for the Duchenne muscular dystrophy pathogenesis are controlled by the dystrophin/nNOS pathway. Cell Metab. 12, 341-351.
Calin, G. A., and Croce, C. M. (2009) Chronic lymphocytic leukemia: interplay between noncoding RNAs and protein-coding genes. Blood 114, 4761-4770.

Calin, G. A., Dumitru, C. D., Shimizu, M., Bichi, R., Zupo, S., Noch, E. et al. (2002). Frequent deletions and down-regulation of micro- RNA genes miR15 and miR16 at 13q14 in chronic lymphocytic leukemia. Proc. Natl. Acad. Sci. U.S.A. 99, 15524-15529.

Cesana, M., Cacchiarelli, D., Legnini, I., Santini, T., Sthandier, O., Chinappi, M., et al. (2011). A long noncoding RNA controls muscle differentiation by functioning as a competing endogenous RNA. Cell 147, 358-369.

Chen, P. S., Su, J. L., Cha, S. T., Tarn, W. Y., Wang, M. Y., Hsu, H. C., et al. (2011). miR-107 promotes tumor progression by targeting the let-7 microRNA in mice and humans. J. Clin. Invest. 121, 3442-3455.

Cortez, M. A., Welsh, J. W., and Calin, G. A. (2012). Circulating 
microRNAs as noninvasive biomarkers in breast cancer. Recent Results Cancer Res. 195, 151-161.

Donnem, T., Fenton, C. G., Lonvik, K., Berg, T., Eklo, K., Andersen, S., et al. (2012). MicroRNA signatures in tumor tissue related to angiogenesis in non-small cell lung cancer. PLOS ONE 7:e29671. doi: 10.1371/journal.pone.0029671

Eaton, G. M., Cody, R. J., Nunziata, E., and Binkley, P. F. (1995). Early left ventricular dysfunction elicits activation of sympathetic drive and attenuation of parasympathetic tone in the paced canine model of congestive heart failure. Circulation $92,555-561$.

Egenvall, A., Bonnett, B. N., Ohagen, P., Olson, P., Hedhammar, A., and Von Euler, H. (2005). Incidence of and survival after mammary tumors in a population of over 80,000 insured female dogs in Sweden from 1995 to 2002. Prev. Vet. Med. 69, 109-127.

Elbashir, S. M., Harborth, J., Lendeckel, W., Yalcin, A., Weber, K., and Tuschl, T. (2001). Duplexes of 21-nucleotide RNAs mediate RNA interference in cultured mammalian cells. Nature 411, 494-498.

Filippov, V., Solovyev, V., Filippova, M., and Gill, S. S. (2000). A novel type of RNase III family proteins in eukaryotes. Gene 245, 213-221.

Fire, A., Xu, S., Montgomery, M. K., Kostas, S. A., Driver, S. E., and Mello, C. C. (1998). Potent and specific genetic interference by doublestranded RNA in Caenorhabditis elegans. Nature 391, 806-811.

Fortin, K. R., Nicholson, R. H., and Nicholson, A. W. (2002). Mouse ribonuclease III. cDNA structure, expression analysis, and chromosomal location. BMC Genomics 3:26. doi: 10.1186/1471-2164-3-26

Fulci, V., Chiaretti, S., Goldoni, M., Azzalin, G., Carucci, N., Tavolaro, S., et al. (2007). Quantitative technologies establish a novel microRNA profile of chronic lymphocytic leukemia. Blood 109, 4944-4951.

Gioia, G., Mortarino, M., Gelain, M. E., Albonico, F., Ciusani, E., Forno, I., et al. (2011). Immunophenotyperelated microRNA expression in canine chronic lymphocytic leukemia. Vet. Immunol. Immunopathol. 142, 228-235.

Gregory, P. A., Bert, A. G., Paterson, E. L., Barry, S. C., Tsykin, A., Farshid, G., et al. (2008). The miR-200 family and miR-205 regulate epithelial to mesenchymal transition by targeting ZEB1 and SIP1. Nat. Cell Biol. 10, 593-601.
Han, J., Lee, Y., Yeom, K. H., Kim, Y. K., Jin, H., and Kim, V. N. (2004). The Drosha-DGCR8 complex in primary microRNA processing. Genes Dev. 18, 3016-3027.

Ibanez-Ventoso, C., Vora, M., and Driscoll, M. (2008). Sequence relationships among C. elegans, $D$. melanogaster and human microRNAs highlight the extensive conservation of microRNAs in biology. PLOS ONE 3:e2818. doi: 10.1371/journal.pone.0002818

Ikeda, K., Mason, P. J., and Bessler, M. (2011). 3'UTR-truncated Hmga2 cDNA causes MPN-like hematopoiesis by conferring a clonal growth advantage at the level of HSC in mice. Blood 117, 5860-5869.

Ionut, V., Liu, H., Mooradian, V., Castro, A. V., Kabir, M., Stefanovski, D., et al. (2008). Novel canine models of obese prediabetes and mild type 2 diabetes. Am. J. Physiol. Endocrinol. Metab. 298, E38-E48.

Jemal, A., Bray, F., Center, M. M., Ferlay, J., Ward, E., and Forman, D. (2011). Global cancer statistics. CA Cancer J. Clin. 61, 69-90.

Kai, Z. S., and Pasquinelli, A. E. (2010). MicroRNA assassins: factors that regulate the disappearance of $\mathrm{miR}$ NAs. Nat. Struct. Mol. Biol. 17, 5-10.

Karlsson, E. K., and Lindblad-Toh, K. (2008). Leader of the pack: gene mapping in dogs and other model organisms. Nat. Rev. Genet. 9 , 713-725.

Khanna, C., Lindblad-Toh, K., Vail, D. London, C., Bergman, P., Barber, L., et al. (2006). The $\operatorname{dog}$ as a cancer model. Nat. Biotechnol. 24, 1065-1066.

Kim, V. N., Han, J., and Siomi, M. C. (2009). Biogenesis of small RNAs in animals. Nat. Rev. Mol. Cell Biol. 10, 126-139.

Kozomara, A., and Griffiths-Jones, S. (2011). miRBase: integrating microRNA annotation and deepsequencing data. Nucleic Acids Res. 39, D152-D157.

Krell, J., Frampton, A. E., Jacob, J., Castellano, L., and Stebbing, J. (2012). miRNAs in breast cancer: ready for real time? Pharmacogenomics 13, 709-719.

Landskroner-Eiger, S., Moneke, I., and Sessa, W. C. (2012). miRNAs as modulators of angiogenesis. Cold Spring Harb. Perspect. Med. 3:a006643. doi: 10.1101/ cshperspect.a006643

Li, W., Duan, R., Kooy, F., Sherman, S. L., Zhou, W., and Jin, P. (2009). Germline mutation of microRNA$125 \mathrm{a}$ is associated with breast cancer. J. Med. Genet. 46, 358-360.
Lin, L., Faraco, J., Li, R., Kadotani, H., Rogers, W., Lin, X., et al. (1999). The sleep disorder canine narcolepsy is caused by a mutation in the hypocretin (orexin) receptor 2 gene. Cell 98, 365-376.

Lindblad-Toh, K., Wade, C. M., Mikkelsen, T. S., Karlsson, E. K. Jaffe, D. B., Kamal, M., et al. (2005). Genome sequence, comparative analysis and haplotype structure of the domestic dog. Nature 438, 803-819.

Lohi, H., Young, E. J., Fitzmaurice, S. N., Rusbridge, C., Chan, E. M., Vervoort, M., et al. (2005). Expanded repeat in canine epilepsy. Science 307, 81.

Lund, E., Guttinger, S., Calado, A., Dahlberg, J. E., and Kutay, U. (2004). Nuclear export of microRNA precursors. Science 303, 95-98.

Mirabello, L., Troisi, R. J., and Savage, S. A. (2009). Osteosarcoma incidence and survival rates from 1973 to 2004: data from the Surveillance, Epidemiology, and end Results Program. Cancer 115, 1531-1543.

Mizuno, H., Nakamura, A., Aoki, Y., Ito, N., Kishi, S., Yamamoto, K., et al. (2011). Identification of muscle-specific microRNAs in serum of muscular dystrophy animal models: promising novel blood-based markers for muscular dystrophy. PLoS ONE 6:e18388. doi: 10.1371/journal.pone.0018388

Mondol, V., and Pasquinelli, A. E. (2012). Let's make it happen: the role of let-7 microRNA in development. Curr. Top. Dev. Biol. 99, 1-30.

Mueller, F., Fuchs, B., and Kaser-Hotz, B. (2007). Comparative biology of human and canine osteosarcoma. Anticancer Res. 27, 155-164.

Noguchi, S., Mori, T., Hoshino, Y., Yamada, N., Maruo, K., and Akao, Y. (2011). MicroRNAs as tumour suppressors in canine and human melanoma cells and as a prognostic factor in canine melanomas. Vet. Comp. Oncol. 9, 1476-5829.

Noguchi, S., Mori, T., Hoshino, Y., Yamada, N., Nakagawa, T., Sasaki, N., et al. (2012a). Comparative study of anti-oncogenic microRNA145 in canine and human malignant melanoma. J. Vet. Med. Sci. 74, 1-8.

Noguchi, S., Mori, T., Otsuka, Y., Yamada, N., Yasui, Y., Iwasaki, J., et al. (2012b). Anti-oncogenic microRNA-203 induces senescence by targeting E2F3 protein in human melanoma cells. J. Biol. Chem. 287, 11769-11777.

Obbard, D. J., Gordon, K. H., Buck, A. H., and Jiggins, F. M. (2009). The evolution of RNAi as a defence against viruses and transposable elements. Philos. Trans. R Soc. Lond. B Biol. Sci. 364, 99-115.

Ohshima, K., Inoue, K., Fujiwara, A., Hatakeyama, K., Kanto, K., Watanabe, Y., et al. (2010). Let-7 microRNA family is selectively secreted into the extracellular environment via exosomes in a metastatic gastric cancer cell line. PLoS ONE 5:e13247. doi: 10.1371/journal.pone.0013247

Olive, V., Jiang, I., and He, L. (2010). mir-17-92, a cluster of miRNAs in the midst of the cancer network. Int. J. Biochem. Cell Biol. 42, 1348-1354.

Ostrander, E. A., Galibert, F., and Patterson, D. F. (2000). Canine genetics comes of age. Trends Genet. $16,117-124$.

Palamarchuk, A., Efanov, A., Nazaryan, N., Santanam, U., Alder, H. Rassenti, L., et al. (2010). 13q14 deletions in CLL involve cooperating tumor suppressors. Blood 115, 3916-3922.

Pan, X., Thompson, R., Meng, X., $\mathrm{Wu}, \mathrm{D}$., and $\mathrm{Xu}, \mathrm{L}$. (2011). Tumortargeted RNA-interference: functional non-viral nanovectors. Am. J. Cancer Res. 1, 25-42.

Paoloni, M., and Khanna, C. (2008). Translation of new cancer treatments from pet dogs to humans. Nat. Rev. Cancer 8, 147-156.

Pasquinelli, A. E., Reinhart, B. J., Slack, F., Martindale, M. Q., Kuroda, M. I., Maller, B., et al. (2000). Conservation of the sequence and temporal expression of let-7 heterochronic regulatory RNA. Nature 408, 86-89.

Pekarsky, Y., Santanam, U., Cimmino, A., Palamarchuk, A., Efanov, A., Maximov, V., et al. (2006). Tcll expression in chronic lymphocytic leukemia is regulated by miR-29 and miR-181. Cancer Res. 66, 11590-11593.

Petrocca, F., and Lieberman, J. (2010). Promise and challenge of RNA interference-based therapy for cancer. J. Clin. Oncol. 29, 747-754.

Pinho, S. S., Carvalho, S., Cabral, J., Reis, C. A., and Gartner, F. (2012). Canine tumors: a spontaneous animal model of human carcinogenesis. Transl. Res. 159, 165-172.

Rofina, J., Van Andel, I., Van Ederen, A. M., Papaioannou, N., Yamaguchi, H., and Gruys, E. (2003). Canine counterpart of senile dementia of the Alzheimer type: amyloid plaques near capillaries but lack of spatial relationship with activated microglia and macrophages. Amyloid 10, 86-96. 
Rossi, S., Shimizu, M., Barbarotto, E., Nicoloso, M. S., Dimitri, F., Sampath, D., et al. (2010). microRNA fingerprinting of CLL patients with chromosome $17 \mathrm{p}$ deletion identify a miR-21 score that stratifies early survival. Blood 116, 945-952.

Rowell, J. L., McCarthy, D. O., and Alvarez, C. E. (2011). Dog models of naturally occurring cancer. Trends Mol. Med. 17, 380-388.

Rybak, A., Fuchs, H., Smirnova, L., Brandt, C., Pohl, E. E., Nitsch, R., et al. (2008). A feedback loop comprising lin-28 and let-7 controls pre-let-7 maturation during neural stem-cell commitment. Nat. Cell Biol. 10, 987-993.

Ryland, G. L., Bearfoot, J. L., Doyle, M. A., Boyle, S. E., Choong, D. Y., Rowley, S. M., et al. (2012). MicroRNA genes and their target 3'-untranslated regions are infrequently somatically mutated in ovarian cancers. PLOS ONE 7:e35805. doi: 10.1371/journal. pone.0035805

Sarver, A. L., Thayanithy, V., Scott, M. C., Cleton-Jansen, A. M., Hogendoorn, P. C., Modiano, J. F., et al. (2013). MicroRNAs at the human $14 \mathrm{q} 32$ locus have prognostic significance in osteosarcoma. Orphanet J. Rare Dis. 8:7. doi: 10.1186/1750-1172-8-7

Sevignani, C., Calin, G. A., Siracusa, L. D., and Croce, C. M. (2006).
Mammalian microRNAs: a small world for fine-tuning gene expression. Mamm. Genome 17, 189-202.

Shan, H., Zhang, Y., Lu, Y., Zhang, Y., Pan, Z., Cai, B., et al. (2009). Downregulation of miR-133 and miR-590 contributes to nicotineinduced atrial remodelling in canines. Cardiovasc. Res. 83, 465-472.

Shore, A. N., Herschkowitz, J. I., and Rosen, J. M. (2012). Noncoding RNAs involved in mammary gland development and tumorigenesis: there's a long way to go. J. Mammary Gland Biol. Neoplasia 17, 43-58.

Sutter, N. B., and Ostrander, E. A. (2004). Dog star rising: the canine genetic system. Nat. Rev. Genet. 5, 900-910.

Tomari, Y., and Zamore, P. D. (2005). Perspective: machines for RNAi. Genes Dev. 19, 517-529.

Uhl, E., Krimer, P., Schliekelman, P., Tompkins, S. M., and Suter, S. (2011). Identification of altered MicroRNA expression in canine lymphoid cell lines and cases of B- and T-Cell lymphomas. Genes Chromosomes Cancer 50, 950-967.

Wang, M., Tan, L. P., Dijkstra, M. K., Van Lom, K., Robertus, J. L., Harms, G., et al. (2008). miRNA analysis in B-cell chronic lymphocytic leukaemia: proliferation centres characterized by low miR-150 and high BIC/miR-155 expression. J. Pathol. 215, 13-20.

Winter, J., Jung, S., Keller, S., Gregory, R. I., and Diederichs, S. (2009) Many roads to maturity: microRNA biogenesis pathways and their regulation. Nat. Cell Biol. 11, 228-234.

Withrow, J. S., and Vail, D. M. (2007). Withrow and MacEwen's Small Animal Clinical Oncology, 4th Edn. St. Louis MO: Saunders Company.

Withrow, J. S., and Vail, D. M. (2012). Withrow and MacEwen's Small Animal Clinical Oncology, 5th Edn. St. Louis MO: Saunders Company.

Wu, H., Xu, H., Miraglia, L. J., and Crooke, S. T. (2000). Human RNase III is a $160-\mathrm{kDa}$ protein involved in preribosomal RNA processing. J. Biol. Chem. 275, 36957-36965.

Yan, K. S., Yan, S., Farooq, A., Han, A. Zeng, L., and Zhou, M. M. (2003). Structure and conserved RNA binding of the PAZ domain. Nature 426, 468-474.

Zeng, Y., and Cullen, B. R. (2006) Recognition and cleavage of primary microRNA transcripts. Methods Mol. Biol. 342, 49-56.

Zhou, D., Li, S., Wen, J., Gong, X., Xu, L., and Luo, Y. (2008) Genome-wide computational analyses of microRNAs and their targets from Canis familiaris. Comput. Biol. Chem. 32, 60-65.
Zhu, D. X., Zhu, W., Fang, C., Fan, L., Zou, Z. J., Wang, Y. H., et al. (2012). miR-181a/b significantly enhances drug sensitivity in chronic lymphocytic leukemia cells via targeting multiple anti-apoptosis genes. Carcinogenesis 33, 1294-1301.

Conflict of Interest Statement: The authors declare that the research was conducted in the absence of any commercial or financial relationships that could be construed as a potential conflict of interest.

Received: 22 August 2012; paper pending published: 04 November 2012; accepted: 13 March 2013; published online: 08 April 2013.

Citation: Wagner S, Willenbrock S, Nolte I and Escobar HM (2013) Comparison of non-coding RNAs in human and canine cancer. Front. Genet. 4:46. doi: 10.3389/ fgene.2013.00046

This article was submitted to Frontiers in Non-Coding RNA, a specialty of Frontiers in Genetics.

Copyright (c) 2013 Wagner, Willenbrock, Nolte and Escobar. This is an open-access article distributed under the terms of the Creative Commons Attribution License, which permits use, distribution and reproduction in other forums, provided the original authors and source are credited and subject to any copyright notices concerning any third-party graphics etc. 